\title{
COVID-19 Infection Clinical Profile, Management, Outcome, and Antibody Response in Kidney Transplant Recipients: A Single Centre Experience
}

\author{
Sanjiv Jasuja $\left(\mathbb{D},{ }^{1}\right.$ Gaurav Sagar $\left(\mathbb{D},{ }^{1}\right.$ Anupam Bahl ${ }^{D},{ }^{1}$ and Shalini Verma ${ }^{2}{ }^{2}$ \\ ${ }^{1}$ Deaprtment of Nephrology, Indraprastha Apollo Hospitals, New Delhi, India \\ ${ }^{2}$ AVATAR Foundation, Department of Clinical Research, New Delhi, India \\ Correspondence should be addressed to Sanjiv Jasuja; sanjivjasuja@yahoo.com
}

Received 14 August 2021; Accepted 13 September 2021; Published 4 October 2021

Academic Editor: Sachin S. Soni

Copyright (c) 2021 Sanjiv Jasuja et al. This is an open access article distributed under the Creative Commons Attribution License, which permits unrestricted use, distribution, and reproduction in any medium, provided the original work is properly cited.

Introduction. Experience of COVID-19 in kidney transplant recipients (KTRs) with clinical presentation, management, factors influencing mortality, and antibody response is limited. Material and Methods. A retrospective data of COVID-19 in KTRs was collected and analyzed. The mortality rate, risk factors, and antibody response were primary objectives, while the clinical presentation, laboratory indicators, and pharmacological management were secondary objectives. Results. The 67 KTRs with polymerase chain reaction (PCR) confirmed COVID-19 infection reported between 1 May 2020 and 31 December 2020; 61.2\% of patients were hospitalized; and $20.9 \%$ needed ventilation. The overall mortality was $26.9 \%$, while blood group A had $50 \%$ mortality. The treatment options and used were steroids (100\%), convalescent plasma (32.8\%), ivermectin (58.2\%), doxycycline $(55.2 \%)$, remdesivir $(34.3 \%)$, tocilizumab $(10.4 \%)$, antibiotics $(61.2 \%)$, anti-fungals $(26.9 \%)$, low molecular weight heparin (45.3\%), and oral anti-coagulants (26.9\%). Anti-nucleosides (mycophenolate or azathioprine) were discontinued in $76.1 \%$ and calcineurin inhibitors $(\mathrm{CNI})$ in $26.9 \%$. Significant mortality $(p<0.001)$ was observed in patients presenting with $\mathrm{SpO}_{2}<94$ needing ICU care, ventilation, dialysis/acute kidney injury (AKI), and empirical therapies like convalescent plasma and remdesivir. The age of survivors versus nonsurvivors was not significantly different $(p=0.02)$. The positive blood culture, low serum albumin, high TLC, high blood urea, interleukin-6, and CT severity score $\geq 15$ were statistically significant in nonsurvivors. Overall mortality, mortality of hospitalized patients, and mortality of ventilated patients was $27 \%$, $44 \%$, and $100 \%$, respectively. The median value of SARS-CoV-2 (COVID-19) IgG antibody was 68.60 (IQR, 28.5-94.25) AU/ml in more than 90\% of survivors. Conclusion. KTRs with COVID-19, needing ICU care, dialysis and ventilation support had poor outcomes. Recovered patients mounted adequate antibody response.

\section{Introduction}

Infection with severe acute respiratory syndrome coronavirus 2 (SARS-CoV-2) mainly affects the respiratory tract but does not spare other organs, brain and kidneys being the major targets. Clinical management of COVID19 infection in kidney transplant recipients (KTRs) calls for early diagnosis and aggressive treatment strategy for a favourable outcome in otherwise reported high mortality group. Kidney injury is well documented with COVID-19, and KTRs are more vulnerable for rapid renal function deterioration. The immunocompromised state, post- transplant duration, age, comorbidities, treatment in a nontransplant centre, and frailty add to the risk factors for poor outcomes $[1,2]$.

The COVID-19 may prove fatal to KTRs with $28 \%$ mortality (range $16 \%-30 \%$ ) $[1,3,4]$ compared to $1 \%-5 \%$ in the general population [5]. The lymphopenia and hypoxemia $\left(\mathrm{SpO}_{2}<94 \%\right)$ at presentation are associated with high mortality in the hospitalized KTRs [2]. Additionally, the KTRs have a high viral load for a longer duration. It is important to monitor these patients until the viral load is negligible by polymerase chain reaction (PCR) to prevent disease spread in the community. 
The exact role of immunosuppression on the progression of COVID-19 is unknown, but it cannot be denied due to associated high mortality. A few recently published case series of COVID-19 in KTRs propose minimizing immunosuppression while continuing steroid therapy [6]. The judicial dose reduction or discontinuation of immunosuppressants needs close supervision.

As experience with COVID-19 in KTRs is limited, there is a need for collective data. We are sharing our experience of KTRs with COVID-19 with a focus on the modifiable and nonmodifiable risk factors, laboratory investigation including inflammatory markers, morbidity profile, response to pharmacological intervention, antibody response, and mortality. The study observations may help in better treatment approach and management of COVID-19 in this population.

\section{Methods}

This retrospective analysis on COVID-19 disease in 67 kidney transplant recipients (KTRs) treated between 1 May 2020 and 31 December 2020 was carried out in the nephrology unit of a tertiary care hospital in New Delhi, India. The aim of this study was to describe the clinical symptoms, risk factors, laboratory profile, disease management, mortality, and antibody response to COVID-19 in KTRs. All the patients were followed up for a minimum of 90 days excluding deaths. Assessing the mortality rate, correlating with risk factors, and IgG antibody response to COVID-19 were the primary objectives; describing the spectrum of clinical presentation, laboratory investigations, and pharmacological management were the secondary objectives.

Data were obtained from the medical records of the hospitals or patient's follow-up submissions. The KTRs with a history of fever, cough, and PCR positive COVID-19 were investigated and managed by a designated COVID-19 treating team in consultation with the treating nephrologist. The diagnosis was based on the guidelines of the World Health Organization [7, 8]. The data on demography, symptoms, laboratory investigations, treatment received, therapeutic outcomes (mortality and recovery), and IgG antibody response were collected. Patients were categorized into obese, overweight, normal, and underweight with body mass index (BMI) criterion calculated as per consensus group recommendation for BMI in Asian population [9].

In all cases at presentation, clinical symptoms and disease severity were recorded as per the Chinese Centre of Disease Control (CDC) [10] criterion by the treating team. The mild disease include nonpneumonia or mild pneumonia (mild symptoms without dyspnoea, respiratory rate $<30$ / min, blood oxygen saturation $\left(\mathrm{SpO}_{2}\right)>93 \%$, and $\mathrm{PaO}_{2} / \mathrm{FiO}_{2}$ ratio $\geq 300 \mathrm{mmHg}$ ). The severe disease had dyspnoea, respiratory rate $\geq 30 / \mathrm{min}, \mathrm{SpO}_{2} \leq 93 \%, \mathrm{PaO}_{2} / \mathrm{FiO}_{2}$ ratio $<300 \mathrm{mmHg}$, and/or lung infiltrates $>50 \%$ within 24 to $48 \mathrm{~h}$ (in our study, $\mathrm{PaO}_{2} / \mathrm{FiO}_{2}$ ratio was not done in domiciliary patients, while chest X-ray at admission was not done in any patient). The definition of critical disease included adult respiratory distress syndrome (ARDS) or respiratory failure, septic shock, and/or multiple organ dysfunction (MOD) or failure $(\mathrm{MOF})$.
In COVID-19, PCR-confirmed KTRs with mild disease and/or having low-grade fever, cough, and/or myalgia were followed up telephonically. These patients were advised to adhere to the domiciliary quarantine guidelines including self-isolation, monitoring body temperature, vitals, oxygen saturation, periodic investigations, and drug treatment as per annexure 1. Patients were advised to consult with a transplant physician or designated COVID-19 physician if there was any worsening in their symptoms and also to discuss and share their laboratory reports at periodic intervals. Schematic representation of the study activities is provided in Figure 1.

Approximately $10 \%$ of completely asymptomatic domiciliary patients were noncompliant for one or all of the prescribed drugs or investigation protocol, but their symptoms and the clinical course was recorded and included in this study. The sustained hypoxia with three consecutive peripheral oxygen saturation readings below $94 \%$ while on room air 60 minutes apart, continuous fever for 3 days, or any haemodynamic instability were the indications for hospitalization.

The demography and symptoms for domiciliary patients were recorded telephonically on first clinical reporting. While for hospitalized patients, these were sourced from triage notes. All patients were evaluated as per unit protocol, summarized in Figure 1. When more than one laboratory parameter values were available, the mean of all available values was used for study corelations.

Post-hospitalization patients were assessed initially at triage, then on regular basis for oxygen requirement in wards. Patients needing $>10$ litre/minute flow of oxygen, by mask or nasal prongs, to maintain oxygen saturation or having other parameters of critical disease as mentioned above were shifted to intensive care unit (ICU) for further treatment. Unless contraindicated, these patients received remdesivir, tocilizumab, and convalescent plasma (two doses). Patients were treated with appropriate anti-microbials depending on clinical condition, white cell counts, blood and urine cultures, infection biomarkers (CRP, procalcitonin trends, and ferritin), and/or radiological imaging. All patients received steroids and oral anti-coagulation or low molecular weight heparin (LMWH) or conventional heparin, unless contraindicated.

The need for respiratory support was proportionate to hypoxia of individual patient, which varied from high flow oxygenation in ward to prone ventilation in intensive care unit (ICU). AKI was defined using the KDIGO-2012 [11] criterion with baseline serum creatinine. The CT scanning was done when patients despite ongoing treatment worsened their oxygen saturation and CT findings were quantified on the basis of CT severity score index [12]. The PCR test was repeated at 15 days frequency till negative on two consecutive days. Post-recovery all patients were subjected to SARS-CoV-2 (COVID-19) IgG quantitative antibody assessment after the availability of test in India as a surrogate for immune response. At the time of this data compilation, anti-spike SARS-CoV-2 IgG antibody test was not routinely available. These tests were conducted 3 weeks to 30 weeks post-onset of COVID-19 infection. 


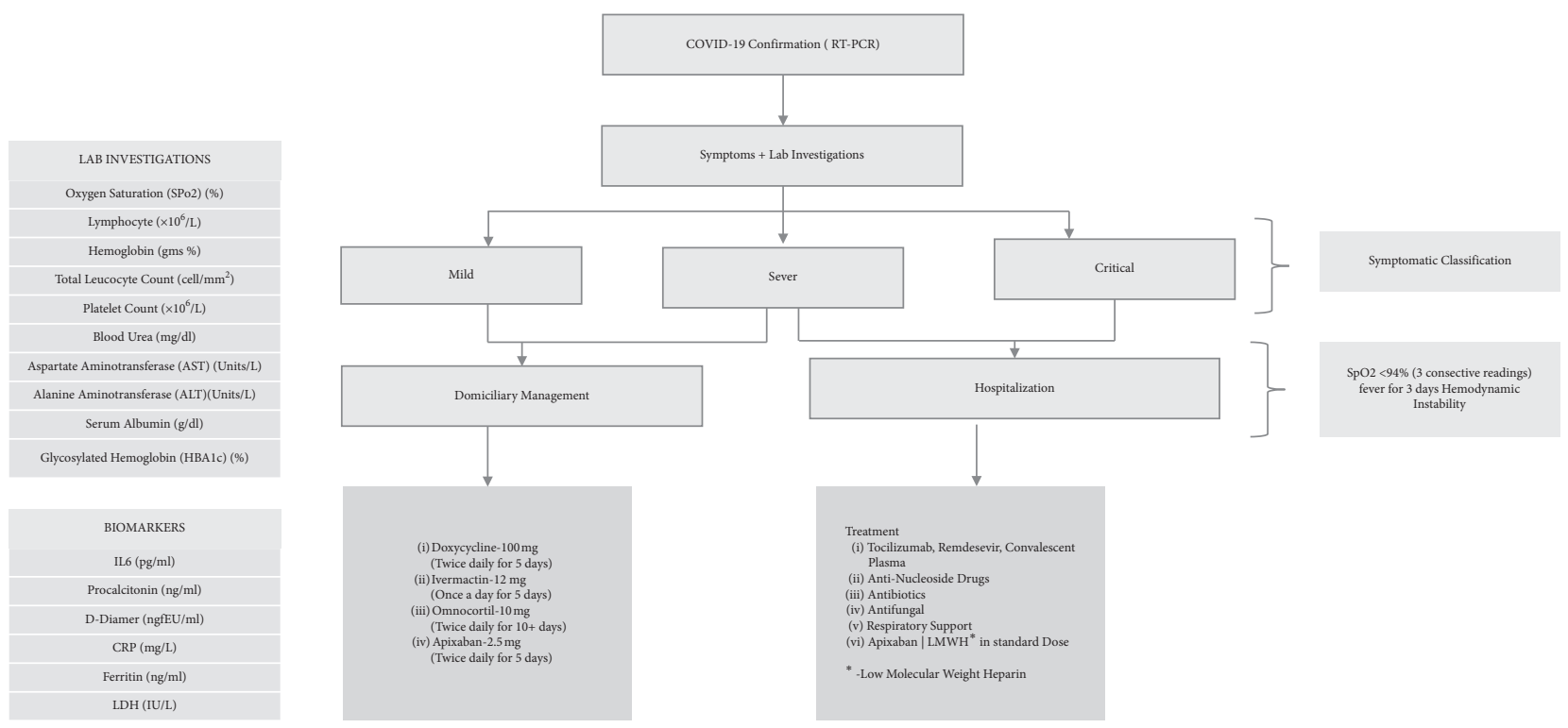

FIgURE 1: Schematic representation of the study in kidney transplant recipients with COVID-19.

2.1. Statistical Analysis. Pooled data was captured on Microsoft Excel and imported to SPSS statistical software version 16.0 (SPSS Inc., Chicago) for analysis, and "boot" package available in R-software version 3.6.1 was applied for determining the median difference confidence interval. Descriptive statistics of continuous variables were summarized as mean \pm standard deviation (SD) and median (interquartile range). The qualitative variables were reported with numbers and percentages. The unpaired Student's $t$-test was applied to compare the mean between survivor and nonsurvivor groups for normally distributed variables; for inflammatory markers and some biomarkers, the nonparametric Mann-Whitney $U$ test was applied due to skewed distribution. The chi-square and Fisher's exact were applied to find the association between mortality and qualitative variables. The mean difference and $95 \%$ confidence interval (CI) were reported for normally distributed variables and median difference and its $95 \%$ confidence interval for skewed variables using the bootstrapping method with 10,000 bootstrapping samples. The binary logistic regression was performed to determine the odds ratio and its $95 \%$ CI. To find the optimal cut-off and discriminate power of biomarkers, receiver operating characteristic (ROC) curve was applied. The Youden index criteria were used for determining the optimal cut-off for the respective biomarker. Multivariable logistic regression was not performed to find the independent risk factors for nonsurvivor due to the small number of cases and missing values of biomarkers, and also some of the variables had zero count. To understand the time to response of $\operatorname{IgG}$ antibodies and sustenance, the various relationships between the antibody values and duration of antibody development from the onset of COVID-19 disease were fitted using curve fitting analysis. The $p$-value less than 0.001 was considered as significant; Bonferroni correction was applied keeping a small sample size in consideration and multiple variable testing. We excluded patients from antibody analysis who had received convalescent plasma during treatment.

\section{Results}

There were 67 kidney transplant recipients (KTRs): males 50 and females 17 (male:female - 1:0.34) with PCR-confirmed COVID-19. The mean (SD) age of patients was 51.34 (13.0) years, range 18-79 years. Mean (SD) BMI was $21(4.21) \mathrm{kg} /$ $\mathrm{m}^{2}$, while $18(26.9 \%)$ and $15(22.4 \%)$ patients were underweight and overweight, respectively (Tables 1 and 2 ).

Lymphopenia was observed in $16(23.8 \%)$ patients. The average post-transplant period (PTP) was 297.25 weeks (see Table 3 for laboratory investigations).

Mean (SD) weight and height were 70.34 (14.99) $\mathrm{kg}$ and 1.67 (0.08) meters, respectively; there was a no statistically significant gender difference in weight: males 71.20 (13.74) $\mathrm{kg}$ and females $57.29(18.59) \mathrm{kg}(p=0.360)$, but it was significant in height: males $1.70(0.07)$ meter and females 1.59 (0.05 meter; $p<0.001)$, which may not be clinically relevant. The blood group distribution among the infected patients were $32.8 \%(n=22), 28.4 \%(n=19), 32.8 \%(n=22)$, and $6 \%(n=4)$ for groups $\mathrm{O}, \mathrm{A}, \mathrm{B}$, and $\mathrm{AB}$, respectively (Table 1).

3.1. Clinical Presentation. Fever $(n=59,88.1 \%)$ and cough ( $n=47,70.1 \%)$ were the major symptoms reported; breathing difficulty $(n=26,38.8 \%)$ and body ache $(n=25,37.3 \%)$ were other main complaints; four patients $(6 \%)$ were detected incidentally with COVID-19. Hypertension (HTN $-n=61,91 \%$ ) and type 2 diabetes mellitus (DM $-n=34,50.7 \%$ ) were the most common comorbidities (Table 1). Concurrent cytomegalovirus (CMV) activation $(n=03,4.5 \%)$, blood culture positivity $(n=05,7.5 \%)$, and urine culture positivity $(n=04,6 \%)$ were observed along with COVID-19.

3.2. Management. Forty-one (61.2\%) patients required hospitalization; only 14 (20.9\%) needed ventilator support; and $\sim 50 \%$ were managed without any respiratory support. 
TABLe 1: Characteristics, comorbidities, and symptoms of kidney transplant recipients with COVID-19.

\begin{tabular}{|c|c|c|c|c|c|c|}
\hline Category & Parameter & $\begin{array}{c}\text { Total } \\
(N=67) \\
n(\%)\end{array}$ & $\begin{array}{l}\text { Survivor } N=49(\% \\
\text { distribution in the } \\
\text { group) }\end{array}$ & $\begin{array}{l}\text { Nonsurvivors } N=18 \\
\text { (\% distribution in the } \\
\text { group) }\end{array}$ & $\begin{array}{l}\text { Odds ratio } \\
(95 \% \mathrm{CI})^{*}\end{array}$ & $P$-value* \\
\hline \multirow{3}{*}{ Gender } & Male & $50(74.6)$ & $37(75.5)$ & $13(72.2)$ & $\begin{array}{c}0.84 \\
(0.25-2.86)\end{array}$ & 0.784 \\
\hline & Female & $17(25.3)$ & $12(24.5)$ & $5(27.8)$ & $1.0(\mathrm{ref})$ & \\
\hline & $\mathrm{O}$ & $22(32.8)$ & $19(38.8)$ & $3(16.7)$ & 1.0 (ref) & \\
\hline \multirow{3}{*}{ Blood group } & A & $19(28.4)$ & $10(20.4)$ & $9(50.0)$ & $\begin{array}{c}5.70 \\
(1.25-25.92)\end{array}$ & 0.024 \\
\hline & $\mathrm{B}$ & $22(32.8)$ & $17(34.7)$ & $5(27.8)$ & $\begin{array}{c}1.86 \\
(0.39-8.99)\end{array}$ & 0.439 \\
\hline & $\mathrm{AB}$ & $4(6.0)$ & $03(6.1)$ & $1(5.6)$ & $\begin{array}{c}2.11 \\
(0.16-27.58)\end{array}$ & 0.569 \\
\hline \multirow{4}{*}{$\begin{array}{l}\text { Body mass index }(\mathrm{kg} / \\
\mathrm{m}^{2)}\end{array}$} & $<18.5$ & $18(26.9)$ & $14(28.6)$ & $4(22.4)$ & 1.00 (ref) & \\
\hline & $18.5-22.9$ & $28(41.8)$ & $22(44.9)$ & $6(33.3)$ & $0.96(0.23-4.0)$ & 0.949 \\
\hline & $23.0-24.9$ & $06(09.0)$ & $02(04.1)$ & $4(22.4)$ & $\begin{array}{c}7.0 \\
(0.92-53.23)\end{array}$ & 0.060 \\
\hline & $\geq 25$ & $15(22.4)$ & $11(22.4)$ & $4(22.4)$ & $\begin{array}{c}1.27 \\
(0.26-6.27)\end{array}$ & 0.767 \\
\hline \multirow{7}{*}{$\begin{array}{l}\text { Pre-existing } \\
\text { comorbidities }\end{array}$} & Diabetes mellitus (DM) & $34(50.7)$ & $23(46.9)$ & $11(61.1)$ & $\begin{array}{c}1.78 \\
(0.59-5.34)\end{array}$ & 0.304 \\
\hline & Hypertension (HTN) & $61(91.0)$ & $43(87.8)$ & $18(100)$ & - & $0.181^{\$}$ \\
\hline & $\begin{array}{c}\text { Chronic liver disease } \\
\text { (CLD) }\end{array}$ & $4(6.0)$ & $2(4.1)$ & $2(11.1)$ & $\begin{array}{c}2.937 \\
(0.38-22.60)\end{array}$ & 0.301 \\
\hline & $\begin{array}{c}\text { Chronic obstructive } \\
\text { airways disease (COAD) }\end{array}$ & $8(11.9)$ & $6(12.2)$ & $2(11.1)$ & $\begin{array}{c}0.896 \\
(0.164-4.90)\end{array}$ & 0.899 \\
\hline & $\begin{array}{c}\text { Vascular disease (CAD/ } \\
\text { PVD) }\end{array}$ & $17(25.6)$ & $9(18.4)$ & $8(44.4)$ & $\begin{array}{c}3.56 \\
(1.10-11.55)\end{array}$ & 0.035 \\
\hline & $\begin{array}{c}\text { Chronic allograft } \\
\text { dysfunction }\end{array}$ & $21(31.3)$ & $14(28.6)$ & $7(38.9)$ & $\begin{array}{c}1.59 \\
(0.51-4.94)\end{array}$ & 0.422 \\
\hline & $\begin{array}{l}\text { Obstructive sleep apnoea } \\
\text { (OSA) }\end{array}$ & $5(7.5)$ & $3(6.1)$ & $2(11.1)$ & $\begin{array}{c}1.92 \\
(0.29-12.53)\end{array}$ & 0.497 \\
\hline \multirow{4}{*}{$\begin{array}{l}\text { Acquired } \\
\text { comorbidities }\end{array}$} & $\begin{array}{c}\text { Cytomegalovirus (CMV) } \\
\text { activation }\end{array}$ & $3(4.5)$ & $0(0.0)$ & $3(16.7)$ & - & $0.017^{\$}$ \\
\hline & Anti-fungal treatment ${ }^{\#}$ & $18(29.9)$ & $4(12.2)$ & $14(77.8)$ & $\begin{array}{c}39.8 \\
(8.70-178.3)\end{array}$ & $<0.001$ \\
\hline & Blood culture, $+\mathrm{ve}^{\# \#}$ & $5(7.5)$ & 0 & $5(27.8)$ & - & $0.001^{\$}$ \\
\hline & Urine culture, $+\mathrm{ve}^{\# \#}$ & $4(6.0)$ & 0 & $4(22.2)$ & - & $0.004^{\$}$ \\
\hline \multirow{3}{*}{$\begin{array}{l}\text { Baseline } \\
\text { immunosuppression }\end{array}$} & CNI (Tac/CyA) & $66(98.5)$ & $48(97.9)$ & $18(100)$ & - & $1.00^{\$}$ \\
\hline & MMF/MPA & $66(98.5)$ & 48 (97.9) & $18(100)$ & - & $1.00^{\$}$ \\
\hline & Steroids & $67(100)$ & $49(100)$ & $18(100)$ & - & - \\
\hline \multirow{7}{*}{ Type of organ } & Living donor & $65(97)$ & $48(97.9)$ & $17(94.4)$ & $\begin{array}{c}0.35 \\
(0.02-5.98)\end{array}$ & $0.467^{\$}$ \\
\hline & Cadaver donor & $2(3.0)$ & $1(2.0)$ & $1(5.6)$ & $\begin{array}{c}2.82 \\
(0.17-47.68)\end{array}$ & 0.472 \\
\hline & Asymptomatic & $4(6.0)$ & $4(8.2)$ & $0(0.0)$ & - & $0.567^{\$}$ \\
\hline & Fever & $59(88.1)$ & $43(87.8)$ & $16(88.9)$ & $\begin{array}{c}1.12 \\
(0.20-6.11)\end{array}$ & 0.899 \\
\hline & Cough & $47(70.1)$ & $36(73.5)$ & $11(61.1)$ & $\begin{array}{c}0.57 \\
(0.18-1.78)\end{array}$ & 0.330 \\
\hline & Sore throat & $18(26.9)$ & $11(22.4)$ & $7(38.9)$ & $\begin{array}{c}2.20 \\
(0.69-7.02)\end{array}$ & 0.184 \\
\hline & Body aches & $25(37.3)$ & $18(36.7)$ & $7(38.9)$ & $\begin{array}{c}1.10 \\
(0.36-3.33)\end{array}$ & 0.872 \\
\hline
\end{tabular}


TABLE 1: Continued.

\begin{tabular}{|c|c|c|c|c|c|c|}
\hline Category & Parameter & $\begin{array}{c}\text { Total } \\
(N=67) \\
n(\%)\end{array}$ & $\begin{array}{c}\text { Survivor } N=49(\% \\
\text { distribution in the } \\
\text { group) }\end{array}$ & $\begin{array}{c}\text { Nonsurvivors } N=18 \\
\text { (\% distribution in the } \\
\text { group) }\end{array}$ & $\begin{array}{l}\text { Odds ratio } \\
(95 \% \mathrm{CI})^{*}\end{array}$ & $P$-value* \\
\hline \multirow{6}{*}{ Symptoms } & Breathing difficulty & $26(38.8)$ & $14(28.6)$ & $12(66.7)$ & $\begin{array}{c}5.00 \\
(1.57-15.94)\end{array}$ & $<0.001$ \\
\hline & $\begin{array}{l}\text { Loss of smell } \\
\text { Distaste }\end{array}$ & $\begin{array}{c}4(6.0) \\
9(13.4)\end{array}$ & $\begin{array}{c}4(8.2) \\
9(18.4)\end{array}$ & $\begin{array}{l}0(0.0) \\
0(0.0)\end{array}$ & $\begin{array}{l}- \\
-\end{array}$ & $\begin{array}{l}0.567^{\$} \\
0.099^{\$}\end{array}$ \\
\hline & Headache & $2(3.0)$ & $1(2.0)$ & $1(5.6)$ & $\begin{array}{c}2.82 \\
(0.17-47.68)\end{array}$ & 0.472 \\
\hline & Loose motions & $5(7.5)$ & $3(6.1)$ & $2(11.1)$ & $\begin{array}{c}1.92 \\
(0.29-12.53)\end{array}$ & 0.497 \\
\hline & Extremes weakness & $8(11.9)$ & $5(10.2)$ & $3(16.7)$ & $\begin{array}{c}1.76 \\
(0.38-8.27)\end{array}$ & 0.474 \\
\hline & Altered sensorium & $3(4.5)$ & $0(0.0)$ & $3(16.7)$ & - & $0.017^{\$}$ \\
\hline
\end{tabular}

${ }^{*}$ Odds ratio could not be computed due to zero count; ${ }^{\$}$ Fisher's exact test; CAD/PVD, coronary artery disease/peripheral vascular disease; ${ }^{*}$ anti-fungal treatment when infection documented by positive urine or blood culture or suspected radiologically during active COVID-19 disease hospitalization or used

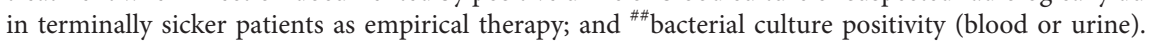

TABLE 2: Laboratory investigations in kidney transplant recipients with COVID-19.

\begin{tabular}{lccc}
\hline Laboratory investigation & Number $(\mathrm{N})$ & Mean $(\mathrm{SD})$ & Min-max \\
\hline Oxygen saturation $\left(\mathrm{SpO}_{2} ; \%\right)$ & 67 & $93.36(5.4)$ & $75-99$ \\
Lymphocyte $\left(\times 10^{9} / \mathrm{L}\right)$ & 64 & $12.68(7.38)$ & $11.14(2.05)$ \\
Haemoglobin $(\mathrm{gm} \%$; $\mathrm{Hb})$ & 67 & $9999(4032)$ & $1.0-45.70$ \\
Total leucocyte count $\left(\mathrm{TLC}\right.$; cells $\left./ \mathrm{mm}^{3}\right)$ & 67 & $203.5(76.5)$ & $6.7-15.25$ \\
Platelet count $\left(\times 10^{9} / \mathrm{L}\right)$ & 67 & $77.27(38.3)$ & $4367-21433$ \\
Blood urea $(\mathrm{mg} / \mathrm{dL})$ & 67 & $2.04(1.31)$ & $37.3-391$ \\
Serum creatinine $(\mathrm{mg} / \mathrm{dL})$ & 66 & $30.32(11.62)$ & $21.06-178.0$ \\
Aspartate aminotransferase $(\mathrm{AST})(\mathrm{IU} / \mathrm{L})$ & 61 & $33.86(18.7)$ & $0.78-6.47$ \\
Alanine aminotransferase $(\mathrm{ALT} ; \mathrm{IU} / \mathrm{L})$ & 62 & $3.55(0.64)$ & $11.5-72.0$ \\
Serum albumin $(\mathrm{gm} / \mathrm{dL})$ & 63 & $7.08(1.58)$ & $8.0-93.5$ \\
Glycosylated haemoglobin $(\mathrm{HBA} 1 \mathrm{c} ; \%)$ & 31 & & $1.8-4.7$ \\
\hline
\end{tabular}

Steroids $(n=67,100 \%)$ were the core treatment. Ivermectin ( $n=39,58.2 \%)$, doxycycline $(n=37,55.2 \%)$, and remdesivir $(n=23,34.3 \%)$ were other coadministered drugs. Convalescent plasma was given to $22(32.8 \%)$ hospitalized patients. Antibiotics and anti-fungals were used in $61.2 \%(n=41)$ and $26.9 \%(n=18)$, respectively, either empirically in terminally sicker patients or based on suggestive imaging or on specific organism grown on culture. Low molecular weight heparin (LMWH; $n=31,45.3 \%)$, oral anti-coagulants $(n=18$, $26.9 \%)$, and anti-platelet agents $(n=02,3.0 \%)$ were prescribed for thromboprophylaxis. Anti-nucleoside drugs were discontinued in 51 patients $(76.1 \%)$; dose reduced in three patients $(4.5 \%)$. Tacrolimus or cyclosporine was continued in same or reduced dose in 49 patients $(73.1 \%)$ and stopped in 18 patients (26.9\%). Thirty-eight percent KTRs (24/63) had AKI; out of these 54.2\% (13/24) needed dialysis, and $66.7 \%(16 / 24)$ died. Computerized tomography (CT) scanning was done in only 30 patients, and out of these, $50 \%$ patients $(n=15)$ had CT severity score $\geq 15$ (Table 4 ).

3.3. Outcome. Forty Patients (59.7\%) recovered completely; nine $(13.4 \%)$ had mild-to-moderate respiratory sequelae after recovery. Eighteen (27\%) patients succumbed to COVID-19 either due to active disease $(15,22.5 \%)$ or due to post-COVID-19 sequelae $(3,4.5 \%)$. The significant mortality $(p<0.001)$ was observed in patients on ventilator support $(14 / 14,100 \%)$ and received convalescent plasma $(n=15 / 18$, $83.3 \%)$ and remdesivir $(n=14 / 18,77.8 \%)$.

Positive blood culture, anti-fungal treatment, higher blood urea, lower serum albumin, and low oxygen saturation at presentation $\left(\mathrm{SpO}_{2}<94\right)$ were significant in nonsurvivors $(p<0.001$; Table 2$)$. The $50 \%$ of nonsurvivors had blood group A (9/18).

The inflammatory markers procalcitonin, CRP, D-dimer, ferritin, and LDH were not statistically significant, while IL-6 levels were significantly higher in nonsurvivors than survivors. The receiver operating characteristics (ROC) curve analysis revealed among the significant inflammatory biomarkers, and IL-6 had maximum discriminatory power (Figure 2). The average duration of active COVID-19 among survivors was 29.43 days.

3.4. Antibody Response. The SARS-CoV-2 (COVID-19) IgG antibodies by chemiluminescence immunoassay (CLIA; LIAISON SARS-CoV-2 S1/S2, DiaSorin, Italy) were measured in 49 patients over 6 months after recovery, while 8 patients had received convalescent plasma during treatment were excluded from final analysis. The median value obtained was 68.6 (interquartile range (IQR), 28.5-94.25) AU/ $\mathrm{ml}$. The $9.8 \%$ patients $(4 / 41)$ had antibody response below 
TABLE 3: Management and treatment administered in kidney transplant recipients with COVID-19.

\begin{tabular}{|c|c|c|}
\hline Parameters & Number & Percentage \\
\hline \multicolumn{3}{|l|}{ Treatment parameters } \\
\hline Hospitalization & 41 & 61.2 \\
\hline Domiciliary & 26 & 38.8 \\
\hline Room air management & 33 & 49.3 \\
\hline Oxygen with mask & 16 & 22.9 \\
\hline Noninvasive ventilator & 5 & 7.5 \\
\hline Ventilator & 14 & 20.9 \\
\hline Steroid & 67 & 100 \\
\hline Azithromycin & 29 & 43.3 \\
\hline HCQS & 5 & 7.5 \\
\hline Ivermectin & 39 & 58.2 \\
\hline Doxycycline & 37 & 55.2 \\
\hline Tocilizumab & 7 & 10.4 \\
\hline Remdesivir & 23 & 34.3 \\
\hline Convalescent plasma & 22 & 32.8 \\
\hline \multicolumn{3}{|l|}{ Thromboprophylaxis } \\
\hline Anti-platelet & 2 & 3 \\
\hline LMWH & 31 & 45.3 \\
\hline $\mathrm{OAC}$ & 18 & 26.9 \\
\hline \multicolumn{3}{|l|}{ Anti-nucleoside drugs } \\
\hline Continued & 10 & 14.9 \\
\hline Dose reduced & 3 & 4.5 \\
\hline Drug stopped & 51 & 76.1 \\
\hline Not taking & 3 & 4.5 \\
\hline \multicolumn{3}{|c|}{ CNI drugs (tacrolimus or cyclosporine) } \\
\hline CNI continued & 48 & 71.6 \\
\hline CNI dose reduced & 1 & 1.5 \\
\hline CNI stopped & 18 & 26.9 \\
\hline \multicolumn{3}{|c|}{ Need for dialysis support (CRRT/SLEDD) } \\
\hline AKI patients needing dialysis* & 13 & 20.6 \\
\hline \multicolumn{3}{|c|}{ Computerized tomographic scanning with CT score $(N=30)$} \\
\hline CT score $<10$ & 09 & 30 \\
\hline CT score $11-14$ & 06 & 20 \\
\hline CT score $\geq 15$ & 15 & 50 \\
\hline ICU requirement & 19 & 28.4 \\
\hline Antibiotics used & 41 & 61.2 \\
\hline Anti-fungal used & 18 & 26.9 \\
\hline
\end{tabular}

HCQS, hydroxy chloroquine sulphate, LMWH, low molecular weight heparin, OAC, oral anti-coagulants, and CNI, calcineurin inhibitors. * Patients with advanced graft dysfunction planned/initiated on HD during pandemic before PCR positivity were excluded.

$12 \mathrm{AU} / \mathrm{ml}$, indicating the absence of SARS-CoV-2 IgG antibodies. The curve fitting analysis found no significant relationship between the antibody values and duration of antibody development from the onset of COVID-19.

\section{Discussion}

Kidney transplant recipients with severe COVID-19 require hospitalization due to rapid disease progression, while the need for intensive care and/or haemodialysis increases the risk of mortality. The disease-associated cytokine release syndrome leads to multiorgan dysfunction including acute kidney injury (AKI) in these patients [13]. The laboratory abnormalities including lymphopenia; elevated acute biochemical markers, that is, C-reactive protein; procalcitonin; interleukin-6; $D$ dimer; and radiological findings of ground glassing; pneumonia; and fibrosis are associated with poor prognosis [14]. The nonmodifiable clinical spectrum associated with poor outcomes includes obesity, pre-existing respiratory disease, hypertension, male gender, age $>60$ years, hypertension, diabetes mellitus, tobacco smoking, pre-existing cardiac diseases, and the first year after transplantation $[3,5,15]$. Elhadedy et al. reported, in their series, that more than $90 \%$ KTRs required hospitalization, and dyspnoea was the chief complaint in $80 \%$ needing ICU service [15].

The mean age of our study population was $>50$ years with male predominance $(75 \%)$ and the median $\left(25^{\text {th }}\right.$ to $75^{\text {th }}$ percentile) of post-transplant duration was 260.0 weeks (IQR, 133.0-390.0 weeks). The predominant comorbidities associated were type 2 diabetes mellitus (51\%), hypertension (91\%), vascular disease (26\%), and chronic allograft dysfunction (31\%). Four asymptomatic cases were detected incidentally while going through COVID-19 screening as a mandatory prerequisite for other ailment hospitalization during the pandemic.

The clinical spectrum of COVID-19 disease in KTRs reported in literature ranged from being asymptomatic to presenting with fever, cough, dyspnoea [2, 15-18], diarrhoea [5], myalgia, chills, fatigue [17], and need for hospitalization (80\%) [16]. The semiology of symptoms in our patients was in line with the literature with fever $(88.1 \%)$, cough $(70.1 \%)$, breathing difficulty $(38.3 \%)$, and body aches (37.3\%). We observed blood groups $\mathrm{O}$ and $\mathrm{B}$ $(32.8 \%$ each) were more affected. The concurrent cytomegalovirus (CMV) activation or infection was observed in $5 \%$ of patients.

The reported incidence of AKI and abnormal renal parameters in COVID-19 patients in the general population is 3\%-9\% [19-22], While the risk of development of AKI in KTRs with COVID-19 is very high (42\%) [23]. We observed in our patients that approximately $40 \%$ of KTRs with COVID-19 developed AKI, more than 50\% of them needed dialysis support, and two-third of the patients succumbed in this group.

The literature has shown significant corelation between various laboratory parameters, clinical spectrum, and outcome. The degree of leucopenia has been associated with disease severity, acute respiratory syndrome (ARDS) [24], and fatal outcome [25, 26]. Similarly, high CRP levels corelates with the severity of COVID-19 [20], ARDS [24], myocardial damage, and mortality [27]. The higher ferritin levels have been associated with ARDS [25] and death [28]. The IL-6, a novel biomarker for COVID-19 severity, has been correlated with mortality in various studies $[21,24]$. In our study, low oxygen saturation at presentation, high blood urea, lower serum albumin, the higher median value of inflammatory marker-IL-6, and high total leucocyte count (TLC) were statistically significant for nonsurvivors (Table 2).

The chest X-ray or computerized tomography (CT scan) assesses the extent of viral pneumonia indicating COVID-19 severity; these changes corelates well with hypoxia and outcome [6]. In our study, a CT scan was done in 30 patients; a severity score of $\geq 15$ was associated with $60 \%$ mortality. 
TABLE 4: Association between mortality and investigations of kidney transplant recipients with COVID-19.

\begin{tabular}{|c|c|c|c|c|}
\hline \multirow{2}{*}{ Parameter } & \multicolumn{2}{|c|}{ Mean (SD) } & \multirow{2}{*}{ Mean difference $(95 \% \mathrm{CI})$} & \multirow{2}{*}{$P$-value* } \\
\hline & Survivors $(n=49)$ & Nonsurvivors $(n=18)$ & & \\
\hline Age (years) & $49.18(13.52)$ & $57.22(9.56)$ & $8.03(1.10-14.98)$ & 0.024 \\
\hline Height (meter) & $1.666(0.08)$ & $1.672(0.08)$ & $0.005(-0.040$ to 0.050$)$ & 0.814 \\
\hline Weight $(\mathrm{kg})$ & $69.18(14.4)$ & $73.5(16.5)$ & $4.32(-3.93$ to 12.56$)$ & 0.3 \\
\hline $\operatorname{BMI}\left(\mathrm{kg} / \mathrm{m}^{2}\right)$ & $20.8(4.23)$ & $21.7(4.17)$ & $0.94(-1.38$ to 3.26$)$ & 0.42 \\
\hline Transplant duration (weeks) & $194(117-370) ; n=49$ & $340(262-448) ; n=18$ & 146 (25.0 to 227.5$)$ & 0.016 \\
\hline Haemoglobin $(\mathrm{gm} \%$; Hb) & $11.46(2.02)$ & $10.27(1.97)$ & $-1.19(-2.29$ to -0.085$)$ & 0.035 \\
\hline Total leucocyte count (cells $/ \mathrm{mm}^{3}$ ) & $8820(2717)$ & $13211(5228)$ & 4391 (2437 to 6345$)$ & $<0.001$ \\
\hline Platelet count $(\mathrm{x} 10(9) / \mathrm{L})$ & $216.8(75.18)$ & $167.0(69.53)$ & $-49.85(-90.44$ to -9.26$)$ & 0.017 \\
\hline Serum creatinine $(\mathrm{mg} / \mathrm{dL})$ & $1.84(1.18)$ & $2.58(1.50)$ & $0.74(0.035$ to 1.44$)$ & 0.04 \\
\hline Blood urea $(\mathrm{mg} / \mathrm{dL})$ & $66.61(32.99)$ & $106.75(37.42)$ & $40.14(20.79$ to 59.48$)$ & $<0.001$ \\
\hline Serum albumin $(\mathrm{gm} / \mathrm{dL})$ & $3.71(0.56)$ & $3.09(0.63)$ & $-0.62(-0.95$ to -0.29$)$ & $<0.001$ \\
\hline Lymphocytes (\%) & $14.02(7.59)$ & $8.98(5.40)$ & $-5.04(-9.05$ to -1.03$)$ & 0.015 \\
\hline Presentation $\mathrm{SpO}_{2}(\%)$ & $95.7(2.78)$ & $86.94(5.77)$ & $-8.77(-10.86$ to -6.68$)$ & $<0.001$ \\
\hline AST (IU/L) & $30.63(11.53)$ & $29.50(12.15)$ & $-1.13(-7.81$ to 5.56$)$ & 0.737 \\
\hline ALT (IU/L) & $35.28(19.60)$ & $29.83(15.90)$ & $-5.55(-16.19$ to 5.09$)$ & 0.301 \\
\hline \multicolumn{5}{|c|}{ Inflammatory markers and other important parameters - median (interquartile range) difference $(95 \% \mathrm{CI})$} \\
\hline IL-6 (pg/ml) & $16.40(4.10-79.10) ; n=29$ & $195.35(64.7-891.4) ; n=14$ & $178.95(50.3$ to 692.2$)$ & $<0.001$ \\
\hline Procalcitonin (ng/ml) & $0.14(0.05-0.48) ; n=29$ & $0.27(0.11-2.84) ; n=15$ & $0.13(-0.06$ to 2.31$)$ & 0.101 \\
\hline D-dimer (ng FEU/ml) & $590(300-1350) ; n=33$ & $1402(712-5563) ; n=16$ & $812(27$ to 4152$)$ & 0.002 \\
\hline CRP $(\mathrm{mg} / \mathrm{L})$ & $34.45(9.55-108.22) ; n=28$ & $149.6(59.6-174.3) ; n=14$ & $115.11(10.0$ to 153.6$)$ & 0.002 \\
\hline Ferritin $(\mathrm{ng} / \mathrm{ml})$ & $522(205-1438) ; n=30$ & $856(715-1243) ; n=16$ & $333.65(-197.0$ to 745.5$)$ & 0.115 \\
\hline $\mathrm{LDH}(\mathrm{IU} / \mathrm{L})$ & $317(257-413) ; n=23$ & $417(291-806) ; n=11$ & $100(-54.0$ to 489.0$)$ & 0.091 \\
\hline Need of dialysis, $n(\%)$ & $3(6.12)$ & $10(55.6)$ & $53.67(10.71-269.04)$ & $<0.001^{\$}$ \\
\hline CT score $\geq 15^{\&}, n(\%)$ & $6(31.6)$ & $9(82.8)$ & $7.17(2.04-25.22)$ & $0.008^{\$}$ \\
\hline Remdesivir & $9(18.4)$ & $14(77.8)$ & $15.56(4.13-58.57)$ & $<0.001^{\$}$ \\
\hline Tocilizumab & $1(2)$ & $6(33.3)$ & $24.0(2.63-218.67)$ & $0.005^{\$}$ \\
\hline Convalescent plasma & $7(14.3)$ & $15(83.3)$ & $30.0(6,86-131.19)$ & $<0.001^{\$}$ \\
\hline Ventilator need & $0(0)$ & $14(77.8)$ & - & $<0.001^{\#}$ \\
\hline Acute kidney injury (AKI), $n(\%)$ & $8(17.78)$ & $16(88.9)$ & $37.0(7.06-194.0)$ & $<0.001^{\$}$ \\
\hline ICU stay, $n(\%)$ & $4(8.2)$ & $15(83.3)$ & $56.3(11.3-280.6)$ & $<0.001^{\$}$ \\
\hline
\end{tabular}

$\mathrm{SpO}_{2}$, oxygen Saturation, Hb, haemoglobin, TLC, total leucocyte count, IL-6, interleukin-6, LDH, lactate dehydrogenase, CRP, C-reactive protein. * Average of all available values. ${ }^{\&} \mathrm{CT}$ score was available for 30,19 survivor and 11 nonsurvivor. ${ }^{\$}$ Odds ratio (95\% confidence interval).

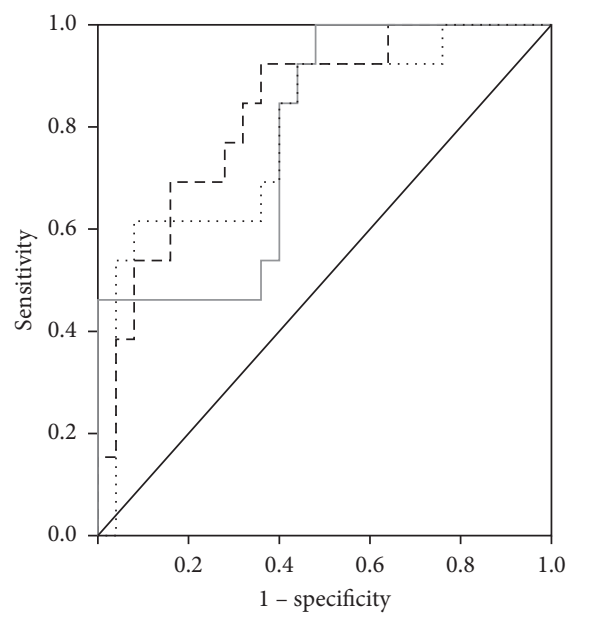

Bio - Markers

- - - IL6

- D Dimmer

Figure 2: ROC curve for diomarkers IL-6, D-dimer, and CRP in kidney transplant recipients with COVID-19.

In published data, mortality of KTRs due to COVID19 is significantly high (KTRs, $24 \%$ vs. $1 \%$ in the general population) [23], particularly those on dialysis for a
The IL6 had maximum area under the ROC curve followed by D Dimmer and CRP. Albeit The area under the ROC curve was not stastistical significant among the biomarkers. The optimal cut-off point for IL6 using Youden index was 47.73 with sensitivity $92.9 \%$ and specificity $65.5 \%$ and for CRP the optimal cut-off was 103 with $61.5 \%$ sensitivity and $84.0 \%$ specificity.

\begin{tabular}{|l|c|c|c|c|c|}
\hline & & & & \multicolumn{2}{|c|}{$\begin{array}{c}\text { Asymptotic 95\% Confidence } \\
\text { Interval }\end{array}$} \\
\hline Test Result Variable (s) & Area & Std. Errora & Asymptotic Sig.b & Lower Bound & Upper Bound \\
\hline IL6 & 0.831 & 0.068 & 0.001 & 0.698 & 0.963 \\
\hline D Dimmer & 0.778 & 0.078 & 0.005 & 0.626 & 0.931 \\
\hline CRP & 0.791 & 0.079 & 0.004 & 0.636 & 0.946 \\
\hline
\end{tabular}

longer time prior to KT [29] and needing hospitalization $[5,15,30]$. Around $61.2 \%$ of our patients required hospitalization; mortality in this group was $44 \%$, while it was 
$27 \%$ overall. Our overall mortality was higher compared to large multicentric pooled data and publications from public sector hospitals from India $(27 \%$ vs. $11.6 \%$ and 9.5\%) [31]. This difference may be due to longer follow-up duration and deaths due to COVID-19 lung sequel. In our study and both of these studies, mortality for ventilated patients was $100 \%$.

In the absence of a definite therapy or guidelines, the modification of immunosuppressants [5] and continuation of steroids along with other COVID-19 management strategies are recommended for KTRs [32]. The other COVID-19 treatment used in KTRs includes anti-virals, hydroxychloroquine, macrolides, remdesivir, tocilizumab, and convalescents plasma as per unit protocol or as per the experience of treating physicians [33]. However, there are equivocal results for COVID-19 outcomes with these drugs in the general population [34-41] and KTRs $[5,12]$. There is a thin line of separation between controlling COVID-19 with these empirical therapies or lowering immunosuppression and their impact on the kidney allograft functioning, directly or by inciting rejection. Hence, one has to decide very diligently on the choice of pharmacotherapy or immunosuppressant dose modification. According to Massachusetts General Hospital COVID-19 treatment guidelines, immunosuppression modification includes a reduction in the dose of calcineurin inhibitors by $50 \%$ and stopping anti-metabolites [42]. Kataria et al. and other authors have outlined the various treatment options including administration of hydroxychloroquine, IL-6 antagonist (tocilizumab), and RNA polymerase inhibitor (remdesivir) along with immunosuppression dose adjustment or withdrawal $[3,16,17]$. Among specific anti-viral therapy, remdesivir has shown good therapeutic result in some studies [43-45]. Convalescent plasma therapy has not been very promising, awaiting more evidence to prove its efficacy, until then its role is limited to severe COVID-19.

In our study, anti-nucleoside drugs (MMF and azathioprine) were stopped or reduced by more than $80 \%$, while CNI was stopped by $27 \%$. The other therapies used in our cohort included steroids (100\%), ivermectin (58.2\%), doxycycline (55.2\%), remdesivir (34.3\%), tocilizumab (10.4\%), and convalescent plasma (32.8\%) as per the unit protocol. The outcome with convalescent plasma was not very promising in our experience as mortality in this group was $63 \%$. The anti-fungal treatment was given based on culture positivity or radiological suggestion or empirical grounds in $30 \%$ of patients. The patients receiving antifungal treatment were significantly high in the nonsurvivor group indicating the adverse prognostic impact of these infections.

The four reported outcomes of COVID-19 in literature in KTRs are: (i) uneventful asymptomatic disease, (ii) complete disease recovery, (iii) recovery with sequelae, and (iv) death (due to active disease or sequelae) [43]. In our study $73 \%$ recovered; $22.5 \%$ died due to active COVID- 19 ; and $4.5 \%$ died due to its sequelae.

Significant mortality was observed in critical patients in our study, on the ventilator, treated with convalescent plasma, dialysis, and remdesivir. As described in methods, these modalities were used in patients not maintaining oxygen saturation or haemodynamic with standard care. Our data do not support the use of remdesivir and plasma therapy in the treatment of COVID-19 as reported in the literature [46].

We observed high mortality associated with blood group A in KTRs. The possible reasons for this observation are not clear. In a meta-analysis of community COVID-19 patients by Nanyang $L$ et al., the individuals with blood group A were more prone to develop the disease with unfavourable outcomes [47]. Interestingly, studies have shown anti-A antibodies inhibit binding of glycosylated SARS-CoV S protein-expressing cells to angiotensin-converting enzyme 2 (ACE2) on the cell surface and hence provide protection by block the interaction between the virus and its receptors; these antibodies missing in blood group A patients. Additionally, the link between blood group A and higher susceptibility of thromboembolism, diabetes, hypertension, recurrent urinary tract infections from Escherichia coli, and gastric ulcers with Helicobacter pylori can also explain high mortality of severe COVID-19 in blood group A [48].

There is limited data available for short- and long-term antibody response to COVID-19 in immunocompromised KTRs $[30,49]$. As per published literature, the duration of up to six months post infection is important to observe the trend of antibodies [50]. An antibody mounting response was noted for more than $90 \%$ of survivors in our study.

The main limitation of our study was that multivariate analyses could not be performed due to the small number of participants and hence the factors associated with mortality in the KTR population could not be assessed; however, Bonferroni correction was applied to keep a small sample size in consideration. We suggest conducting a statistically powered study to understand this antibody trend in KTRs and overall outcomes. The other limitation is only KTR were included in the study, so findings are not applicable to other organ transplants. The level of protection and duration of persistence of these antibodies and the efficacy of the vaccine is still not clear; these queries will settle over a period of time with a better understanding of COVID-19. With the vaccine for COVID-19 being available, KT recipients must be prioritized for vaccination, which may bring down the infection rate, associated complications, disease severity, and mortality.

\section{Conclusion}

Hospitalization, AKI, high CT score, activation of opportunistic infections, need for ventilation and dialysis, treatment with convalescent plasma, and remdesivir were associated with higher mortality due to COVID-19 in KTRs. Out of biomarkers, IL-6 correlates significantly with mortality. Half of the patients in the mortality group had blood group $\mathrm{A}$. The IgG antibody response was noted post-COVID-19 in more than $90 \%$ of patients. Immunosuppression should be tailored by either 
discontinuation or dose reduction of anti-nucleosides and CNI, while high dose steroids can compensate immunosuppression reduction with the contribution to the outcome $[51,52]$.

\section{Data Availability}

All the data are well verified and authentic and available on request; please contact Dr. Sanjiv Jasuja at sanjivjasuja@ yahoo.com.

\section{Conflicts of Interest}

There are no conflicts of interest associated with this manuscript.

\section{Acknowledgments}

The authors profusely acknowledge the support extended by Dr. Santhosh Varughese, Professor and Head, Department of Nephrology, CMC, Vellore, to critically review the manuscript, Dr. N. Subramanian, Director, Medical Services, IAH, for granting them the permissions to do the entire exercise, Dr. Rajeev Kumar Malhotra for the statistical analysis, and Dr. Latha MS for editing the manuscript. The authors indeed acknowledge the assistance provided by Mr. Nirmal Maseeh and Mr. Santosh Singh for data compilation and Mr. Ravi Kumar and Mr. Ashwani Gupta for the graphical inputs.

\section{References}

[1] L. B. Hilbrands, R. Duivenvoorden, P. Vart et al., "COVID-19related mortality in kidney transplant and dialysis patients: results of the ERACODA collaboration," Nephrology Dialysis Transplantation: Official Publication of the European Dialysis and Transplant Association-European Renal Association, vol. 35, pp. 1973-1983, 2020.

[2] V. Nair, N. Jandovitz, and M. Abate, "Risk factors for mortality in kidney transplant recipients with COVID-19," in Proceedings of the Kidney Week 2020 Reimagined Virtual Conference, October 2020, https://pesquisa.bvsalud.org/global-literature-on-novelcoronavirus-2019-ncov/resource/pt/covidwho-984635.

[3] A. Kataria, I. Yakubu, R. Winstead, M. Gowda, and G. Gupta, "COVID-19 in kidney transplantation: epidemiology, management considerations, and the impact on kidney transplant practice," Transplantation Direct, vol. 6, no. 8, Article ID e582, 2020.

[4] S. Bell, J. Campbell, J. Campbell et al., "COVID-19 in patients undergoing chronic kidney replacement therapy and kidney transplant recipients in Scotland: findings and experience from the Scottish renal registry," BMC Nephrology, vol. 21, no. 1, p. 419, 2020

[5] E. Akalin, Y. Azzi, R. Bartash et al., "Covid-19 and kidney transplantation," New England Journal of Medicine, vol. 382, no. 25, pp. 2475-2477, 2020.

[6] D. Banerjee, J. Popoola, S. Shah, I. C. Ster, V. Quan, and M. Phanish, "COVID-19 infection in kidney transplant recipients," Kidney International, vol. 97, no. 6, pp. 1076-1082, 2020.

[7] World Health Organization, Coronavirus Disease (COVID19) Advice for the Public, WHO, Geneva, Switzerland, 2021, https://www.who.int/emergencies/diseases/novelcoronavirus-2019/advice-for-public.

[8] World Health Organization, Country and Technical Guidance, WHO, Geneva, Switzerland, 2020, https://www.who.int/ emergencies/diseases/novel-coronavirus-2019/technicalguidance.

[9] A. Misra, P. Chowbey, B. M. Makkar et al., "Consensus statement for diagnosis of obesity, abdominal obesity and the metabolic syndrome for Asian Indians and recommendations for physical activity, medical and surgical management," The Journal of the Association of Physicians of India, vol. 57, pp. 163-170, 2009.

[10] M. Francone, F. Iafrate, G. M. Masci et al., "Chest CT score in COVID-19 patients: correlation with disease severity and short-term prognosis," European Radiology, vol. 30, no. 12, pp. $6808-6817,2020$.

[11] K. M. Andrassy, "Comments on 'KDIGO 2012 clinical practice guideline for the evaluation and management of chronic kidney disease," Kidney International, vol. 843, p. 622, 623.

[12] A. M. Al-Mosawe, H. m. Abdulwahid, and N. A. H. Fayadh, "Spectrum of CT appearance and CT severity index of COVID-19 pulmonary infection in correlation with age, sex, and PCR test: an Iraqi experience," Egyptian Journal of Radiology and Nuclear Medicine, vol. 52, no. 1, p. 40, 2021.

[13] J. B. Moore and C. H. June, "Cytokine release syndrome in severe COVID-19," Science, vol. 368, no. 6490, pp. 473-474, 2020.

[14] J. González and G. Ciancio, "Early experience with COVID-19 in kidney transplantation recipients: update and review," International Brazilian Journal of Urology: Official Journal of the Brazilian Society of Urology, vol. 46, no. suppl.1, pp. 145-155, 2020.

[15] M. A. Elhadedy, Y. Marie, and A Halawa, "COVID-19 in renal transplant recipients: case series and a brief review of current evidence," Nephron, vol. 145, no. 2, pp. 192-198, 2021.

[16] A. Devresse, L. Belkhir, B. Vo et al., "COVID-19 infection in kidney transplant recipients: a single-center case series of 22 cases from Belgium," Kidney Medicine, vol. 2, no. 4, pp. $459-466,2020$.

[17] V. Nair, N. Jandovitz, J. S. Hirsch et al., "COVID-19 in kidney transplant recipients," American Journal of Transplantation, vol. 20, no. 7, pp. 1819-1825, 2020.

[18] M. Oltean, J. M. Søfteland, J. Bagge et al., "Covid-19 in kidney transplant recipients: a systematic review of the case series available three months into the pandemic," Infectious Diseases, vol. 52, no. 11, pp. 830-837, 2020.

[19] W.-j. Guan, Z.-y. Ni, Y. Hu et al., "Clinical characteristics of coronavirus disease 2019 in China," New England Journal of Medicine, vol. 382, no. 18, pp. 1708-1720, 2020.

[20] D. Wang, B. Hu, C. Hu et al., "Clinical characteristics of 138 hospitalized patients with 2019 novel coronavirus-infected pneumonia in Wuhan, China," Journal of the American Medical Association, vol. 323, no. 11, pp. 1061-1069, 2020.

[21] N. Chen, M. Zhou, X. Dong et al., "Epidemiological and clinical characteristics of 99 cases of 2019 novel coronavirus pneumonia in Wuhan, China: a descriptive study," The Lancet, vol. 395, no. 10223, pp. 507-513, 2020.

[22] Y. Cheng, R. Luo, K. Wang et al., "Kidney disease is associated with in-hospital death of patients with COVID-19," Kidney International, vol. 97, no. 5, pp. 829-838, 2020.

[23] M. Elias, D. Pievani, C. Randoux et al., "COVID-19 infection in kidney transplant recipients: disease incidence and clinical 
outcomes," Journal of the American Society of Nephrology, vol. 31, no. 10, pp. 2413-2423, 2020.

[24] C. Wu, X. Chen, Y. Cai et al., "Risk factors associated with acute respiratory distress syndrome and death in patients with coronavirus disease 2019 pneumonia in Wuhan, China," JAMA Internal Medicine, vol. 180, no. 7, pp. 934-943, 2020.

[25] Y. Deng, W. Liu, K. Liu et al., "Clinical characteristics of fatal and recovered cases of coronavirus disease 2019 in Wuhan, China: a retrospective study," Chinese Medical Journal, vol. 133, no. 11, pp. 1261-1267, 2020.

[26] C. Qin, L. Zhou, Z. Hu et al., "Dysregulation of immune response in patients with coronavirus 2019 (COVID-19) in Wuhan, China," Clinical Infectious Diseases, vol. 71, no. 15, pp. 762-768, 2020.

[27] S. Shi, M. Qin, B. Shen et al., "Association of cardiac injury with mortality in hospitalized patients with COVID-19 in Wuhan, China," JAMA Cardiology, vol. 5, no. 7, pp. 802-810, 2020.

[28] F. Zhou, T. Yu, R. Du et al., "Clinical course and risk factors for mortality of adult inpatients with COVID-19 in Wuhan, China: a retrospective cohort study," The Lancet, vol. 395, no. 10229, pp. 1054-1062, 2020.

[29] C. M. Hsu and D. E. Weiner, "COVID-19 in dialysis patients: outlasting and outsmarting a pandemic," Kidney International, vol. 98, no. 6, pp. 1402-1404, 2020.

[30] Y. Azzi, M. Parides, O. Alani et al., "COVID-19 infection in kidney transplant recipients at the epicenter of pandemics," Kidney International, vol. 98, no. 6, pp. 1559-1567, 2020.

[31] V. B. Kute, A. K. Bhalla, S. Guleria et al., "Clinical profile and outcome of COVID-19 in 250 kidney transplant recipients: a multicenter cohort study from India," Transplantation, vol. 105 , no. 4 , pp. 851-860, 2021.

[32] E. G. Oguz, K. G. Atilgan, S. G. Cimen et al., "COVID-19 infection in a kidney transplant recipient-special emphasis on pharmacokinetic interactions: a case report," World Journal of Transplantation, vol. 10, no. 11, pp. 365-371, 2020.

[33] A. Bhimraj, R. L. Morgan, A. H. Shumaker et al., "Infectious diseases society of America guidelines on the treatment and management of patients with COVID-19," Infectious Diseases Society of America, In press, 2021, https://pubmed.ncbi.nlm. nih.gov/32338708/.

[34] C. A. Devaux, J.-M. Rolain, P. Colson, and D. Raoult, "New insights on the antiviral effects of chloroquine against coronavirus: what to expect for COVID-19?" International Journal of Antimicrobial Agents, vol. 55, no. 5, Article ID 105938, 2020.

[35] Multicenter collaboration group of Department of Science and Technology of Guangdong Province and Health Commission of Guangdong Province for Chloroquine in the Treatment of Novel Coronavirus Pneumonia, "Expert consensus on chloroquine phosphate for the treatment of novel coronavirus pneumonia," Zhonghua Jiehe He Huxi Zazhi, vol. 43, pp. 185-188, 2020.

[36] M. L. Holshue, C. DeBolt, S. Lindquist et al., "First case of 2019 novel coronavirus in the United States," New England Journal of Medicine, vol. 382, no. 10, pp. 929-936, 2020.

[37] C.-C. Lu, M.-Y. Chen, W.-S. Lee, and Y.-L. Chang, "Potential therapeutic agents against COVID-19: what we know so far," Journal of the Chinese Medical Association, vol. 83, no. 6, pp. 534-536, 2020.

[38] C. Chen, Y. Zhang, J. Huang et al., "Favipiravir versus Arbidol for COVID-19: a randomized clinical trial,” medRxiv, vol. 3, Article ID 20037432, 2020.
[39] J. H. Beigel, K. M. Tomashek, L. E. Dodd et al., "Remdesivir for the treatment of covid-19-final report," New England Journal of Medicine, vol. 383, no. 19, pp. 1813-1826, 2020.

[40] Y. Wang, D. Zhang, G. Du et al., "Remdesivir in adults with severe COVID-19: a randomised, double-blind, placebocontrolled, multicentre trial," The Lancet, vol. 395, no. 10236, pp. 1569-1578, 2020.

[41] K. Duan, B. Liu, C. Li et al., "Effectiveness of convalescent plasma therapy in severe COVID-19 patients," Proceedings of the National Academy of Sciences, vol. 117, no. 17, pp. 9490-9496, 2020.

[42] V. Kute, S. Varugese, N. Prasad, S. Shroff, and S. K. Agarwal, "On behalf of COVID 19 working group of Indian society of nephrology. Renal transplant guidelines with reference to COVID-19 infection," Indian Journal of Nephrology, vol. 30, pp. 176-178, 2020.

[43] R. T. Eastman, J. S. Roth, K. R. Brimacombe et al., "Remdesivir: a review of its discovery and development leading to emergency use authorization for treatment of COVID-19," ACS Central Science, vol. 6, no. 5, pp. 672-683, 2020.

[44] T. P. Sheahan, A. C. Sims, R. L. Graham et al., "Broadspectrum antiviral GS-5734 inhibits both epidemic and zoonotic coronaviruses," Science Translational Medicine, vol. 9, no. 396, Article ID eaal3653, 2017.

[45] T. P. Sheahan, A. C. Sims, S. R. Leist et al., "Comparative therapeutic efficacy of remdesivir and combination lopinavir, ritonavir, and interferon beta against MERS-CoV," Nature Communications, vol. 11, no. 1, p. 222, 2020.

[46] E. K. McCreary and D. C. Angus, "Efficacy of remdesivir in COVID-19," Journal of the American Medical Association, vol. 324, no. 11, pp. 1041-1042, 2020.

[47] N. Liu, T. Zhang, L. Zhang et al., "The impact of ABO blood group on COVID-19 infection risk and mortality: a systematic review and meta-analysis," Blood Reviews, vol. 48, Article ID 100785, 2021.

[48] S. Zhou and I. Welsby, "Is ABO blood group truly a risk factor for thrombosis and adverse outcomes?" World Journal of Cardiology, vol. 6, no. 9, p. 985, 2014.

[49] M. D. Fainareti Zervou, N. Ali, H. J. Neumann, R. Pellett Madan, and S. A. Mehta, "COVID-19 antibody responses in solid organ transplant recipients," Open Forum Infectious Diseases, vol. 7, no. Supplement_1, p. S332, 2020.

[50] I. Benotmane, G. G. Vargas, and A. Velay, "Persistence of SARS-CoV-2 antibodies in kidney transplant recipients, letter to editor," American Journal of Transplantation, vol. 21, no. 6, pp. 2307-2310, 2021.

[51] V. Dahl Mathiasen, S. Jensen-Fangel, K. Skov, and S. Leth, "Uneventful case of COVID-19 in a kidney transplant recipient," BMJ Case Reports, vol. 13, no. 7, Article ID e237427, 2020.

[52] A. B. B. Laguipo, "Blood types and COVID-19 risk confirmed," New England Journal of Medicine, 2020, https:// www.news-medical.net/news/20200618/Blood-types-andCOVID-19-risk-confirmed.aspx. 